\title{
INVERSION AND REPRESENTATION FOR THE POISSON-LAGUERRE TRANSFORM
}

DEBORAH TEPPER HAIMO

ABSTRACT. The Poisson-Laguerre transform of a function $\phi$ is given by

$$
u(n, t)=\sum_{m=0}^{\infty} g(n, m ; t) \phi(m) \frac{m !}{\Gamma(m+\alpha+1)}
$$

where $g$, defined by

$$
\begin{aligned}
g(n, m ; t)= & \frac{\Gamma(n+m+\alpha+1)}{n ! m !} \frac{t^{m+m}}{(1+t)^{n+m+\alpha+1}} \\
& \cdot{ }_{2} F_{1}\left(-n,-m ;-n-m-\alpha ; 1-\frac{1}{t^{2}}\right),
\end{aligned}
$$

is the associated function of the source solution $g(n ; t)=g(n, 0 ; t)$ of the Laguerre difference heat equation

$$
\nabla_{n} u(n, t)=u_{t}(n, t)
$$

with

$$
\nabla_{n} f(n)=(n+1) f(n+1)=(2 n+\alpha+1) f(n)+(n+\alpha) f(n-1) .
$$

A simple algorithm for the inversion of the transform $(*)$ is derived. For $m=0$, the transform (*) is basically a power series so that the inversion algorithm leads to a useful identity involving binomial coefficients. In addition, a subclass of functions is characterized that is representable by a PoissonLaguerre transform $(*)$.

1. Introduction. The Laguerre difference heat equation is given by

$$
\nabla_{n} u(n, t)=\frac{\partial}{\partial t} u(n, t),
$$

where we define the Laguerre difference operator $\nabla_{n}$ by

$$
\nabla_{n} f(n)=(n+1) f(n+1)-(2 n+1+\alpha) f(n)+(n+\alpha) f(n-1), \quad \alpha \geq 0 .
$$

The fundamental solution of equation (1.1) is the function

$$
\begin{aligned}
g(n ; t) & =\int_{0}^{\infty} e^{-t x} L_{n}^{\alpha}(x) d \Omega(x), \quad d \Omega(x)=e^{-x} x^{\alpha} d x, t>-1, \\
& =\frac{1}{\rho(n)} \frac{t^{n}}{(1+t)^{n+\alpha+1}}, \quad \rho(n)=\frac{n !}{\Gamma(n+\alpha+1)},
\end{aligned}
$$

where $L_{n}^{\alpha}(x)$ is the Laguerre polynomial of degree $n$. See $[4,(7)$, p. 188].

Received by the editors August 9, 1983.

1980 Mathematics Subject Classification. Primary 44A15; Secondary 35Q99, 39A10. 
The associated function of $g(n ; t)$ is given by

$$
\begin{aligned}
g(n, m ; t)= & \int_{0}^{\infty} e^{-t x} L_{n}^{\alpha}(x) L_{m}^{\alpha}(x) d \Omega(x), \quad t>-1, \\
= & \frac{\Gamma(n+m+\alpha+1)}{n ! m !} \frac{t^{n+m}}{(1+t)^{n+m+\alpha+1}} \\
& \cdot{ }_{2} F_{1}\left(-n,-m ;-n-m-\alpha ; 1-\frac{1}{t^{2}}\right) .
\end{aligned}
$$

See $[2,(2.28)$, p. 201].

The Poisson-Laguerre transform of a function $\varphi$ defined for $n=0,1,2, \ldots$ is

$$
u(n, t)=\sum_{m=0}^{\infty} g(n, m ; t) \varphi(m) \rho(m)
$$

whenever the series converges, as given in Definition 2.13 of $[\mathbf{2}$, p. 201].

Our goal is to derive a simple algorithm for the inversion of the Poisson-Laguerre transform (1.5). Since the reduced Poisson-Laguerre transform, obtained when $n=0$ in (1.5), is basically a power series, the algorithm leads to a useful identity involving binomial coefficients. In addition, we characterize a subclass of functions representable by a Poisson-Laguerre transform.

The results provide a discrete analogue, in part, of those for the corresponding transforms for the classical heat equation $[\mathbf{6}, \mathbf{7}]$, for the generalized heat equation $[\mathbf{1}, \mathbf{3}]$, and of the Laguerre differential heat equation $[\mathbf{5}]$.

2. Preliminary results. Let $T$ be the set $\{-1,0,1, \ldots\} \times\{-\infty<t<\infty\}$ of vertical lines in the plane and let $S$ be a subset of $T$. A point $P=(n, t) \in S$ is an inner point of $S$ if there is a neighborhood $N$ of $t$ such that $\{(n, t) \mid t \in N\} \subset S$ and if the points $(n-1, t),(n+1, t) \in S . S$ is a domain if its vertical segments are connected open sets and if each of its vertical segments, except the end segments, has an inner point.

A function $u(n, t)$ in a domain $S$ of $T$ is a Laguerre temperature if and only if $u(n, t) \in C^{1}$ as a function of $t$ and $u(n, t)$ satisfies the Laguerre difference heat equation (1.1) for every inner point of $S$.

In [2] we established that a convergent Poisson-Laguerre transform (1.5) is a Laguerre temperature $u(n, t)$ with initial temperature $u(n, 0+)=\varphi(n)$. We note, then, that the reduced Poisson-Laguerre transform $u(0, t)$ may be interpreted as a Laguerre temperature at $n=0 t$ seconds after the initial Laguerre temperature is $\varphi(n)$. We will exploit this approach in deriving an inversion algorithm. To this end, we need the following series representation for the associated source solution.

LEMMA. For $t>-1$,

$$
g(n, m ; t)=\sum_{k=0}^{m} \frac{\Gamma(m+\alpha+1)}{k !(m-k) ! \Gamma(k+\alpha+1)}\left(\frac{\partial}{\partial t}\right)^{k} g(n ; t)
$$


Proof. We have, using the definition of the Laguerre polynomial,

$$
\begin{aligned}
g(n, m ; t) & =\int_{0}^{\infty} e^{-t x} L_{n}^{\alpha}(x) L_{m}^{\alpha}(x) d \Omega(x) \\
& =\int_{0}^{\infty} e^{-t x} L_{n}^{\alpha}(x) d \Omega(x) \sum_{k=0}^{m} \frac{\Gamma(m+\alpha+1)(-x)^{k}}{k ! \Gamma(k+\alpha+1)(m-k) !} \\
& =\sum_{k=0}^{m} \frac{\Gamma(m+\alpha+1)}{k ! \Gamma(k+\alpha+1)(m-k) !} \int_{0}^{\infty}(-x)^{k} e^{-t x} L_{n}^{\alpha}(x) d \Omega(x) \\
& =\sum_{k=0}^{m} \frac{\Gamma(m+\alpha+1)}{k ! \Gamma(k+\alpha+1)(m-k) !}\left(\frac{\partial}{\partial t}\right)^{k} g(n ; t),
\end{aligned}
$$

where the last equation results from the validity of bringing the operator $(\partial / \partial t)^{k}$ under the integral sign in $(1.3), g(n ; t)$ being a Laplace transform. See $[8$, Theorem 5a, p. 57].

3. Inversion. We now may establish our primary inversion algorithm.

THEOREM 3.1. For given $\sigma$, let

$$
u(n, t)=\sum_{m=0}^{\infty} g(n, m ; t) \varphi(m) \rho(m)
$$

converge for $0<t<\sigma$. Then

$$
\varphi(n)=\lim _{t \rightarrow 0^{+}} \sum_{k=0}^{n} \frac{\Gamma(n+\alpha+1)}{k !(n-k) ! \Gamma(k+\alpha+1)} u^{(k)}(0, t) .
$$

Proof. Consider

$$
\begin{aligned}
u(n, t) & =\sum_{m=0}^{\infty} g(n, m ; t) \varphi(m) \rho(m) \\
& =\sum_{k=0}^{n} \frac{\Gamma(n+\alpha+1)}{k ! \Gamma(k+\alpha+1)(n-k) !} \sum_{m=0}^{\infty} \varphi(m)\left(\frac{\partial}{\partial t}\right)^{k} g(m ; t) \rho(m) \\
& =\sum_{k=0}^{n} \frac{\Gamma(n+\alpha+1)}{k ! \Gamma(k+\alpha+1)(n-k) !} u^{(k)}(0, t),
\end{aligned}
$$

where the second equation follows from (2.1) and the last from the validity of bringing the derivative $(d / d t)^{k}$ within the summation sign of $(3.1)$. The result then follows on appealing to the inversion of the Poisson-Laguerre transform (3.3) as established in $\left[2\right.$, Theorem 6.1, p. 211], whereupon $\lim _{t \rightarrow 0^{+}} u(n, t)=\varphi(n)$.

Letting $n=0$ in (3.1), we have the inversion of the reduced Poisson-Laguerre transform given by the following.

COROLlaRY 3.2. Let

$$
f(t)=\sum_{m=0}^{\infty} g(m ; t) \varphi(m) \rho(m)
$$


converge for $0<t<\sigma$. Then

$$
\varphi(n)=\lim _{t \rightarrow 0^{+}} \sum_{k=0}^{n} \frac{\Gamma(n+\alpha+1)}{k !(n-k) ! \Gamma(k+\alpha+1)} f^{(k)}(t) .
$$

Note that using (1.3) in (3.4), we find that

$$
f(t)=\frac{1}{(1+t)^{\alpha+1}} \sum_{m=0}^{\infty}\left(\frac{t}{1+t}\right)^{m} \varphi(m)
$$

which gives a power series representation in $t /(1+t)$ for $(1+t)^{\alpha+1} f(t)$. The coefficients $\varphi(n)$ are then given by

$$
\begin{aligned}
\varphi(n) & =\left.\frac{1}{n !} \frac{d^{n}}{d t^{n}}\left(\frac{1}{(1-t)^{\alpha+1}} f\left(\frac{t}{1+t}\right)\right)\right|_{t=0} \\
& =\sum_{k=0}^{n} \frac{1}{k !(n-k) !} \frac{\Gamma(n+\alpha+1-k)}{\Gamma(\alpha+1)} \sum_{l=0}^{k}\left(\begin{array}{c}
k \\
l
\end{array}\right) \frac{(k-1) !}{(l-1) !} f^{(l)}(0) \\
& =\sum_{l=0}^{n} \frac{f^{(l)}(0)}{l !(l-1) !} \sum_{k=l}^{n} \frac{(k-1) ! \Gamma(n+\alpha+1-k)}{(k-l) !(n-k) ! \Gamma(\alpha+1)} .
\end{aligned}
$$

Comparing (3.7) with (3.5), we have the following identity.

COROLlary 3.3. For $l, n=0,1,2, \ldots$,

$$
\left(\begin{array}{c}
n+\alpha \\
l+\alpha
\end{array}\right)=\sum_{k=l}^{n}\left(\begin{array}{c}
n+\alpha-k \\
\alpha
\end{array}\right)\left(\begin{array}{c}
k-1 \\
l-1
\end{array}\right) .
$$

Corollary 3.2 can be used to invert the Laguerre transform as in the following.

THEOREM 3.4. Let

$$
\widehat{\varphi}(x)=\sum_{n=0}^{\infty} L_{n}^{\alpha}(x) \varphi(n) \rho(n) \quad \text { with } \sum_{n=0}^{\infty}|\varphi(n)|<\infty .
$$

Then

$$
\varphi(n)=\lim _{t \rightarrow 0^{+}} \sum_{k=0}^{n} \frac{\Gamma(n+\alpha+1)}{k !(n-k) ! \Gamma(k+\alpha+1)} R^{(k)}(t),
$$

where

$$
R(t)=\int_{0}^{\infty} e^{-t u} \widehat{\varphi}(u) d \Omega(u) .
$$

Proof. We have

$$
\begin{aligned}
R(t) & =\int_{0}^{\infty} e^{-t u} d \Omega(u) \sum_{n=0}^{\infty} L_{n}^{\alpha}(u) \varphi(n) \rho(n) \\
& =\sum_{n=0}^{\infty} \varphi(n) \rho(n) \int_{0}^{\infty} e^{-t u} L_{n}^{\alpha}(u) d \Omega(u) \\
& =\sum_{n=0}^{\infty} g(n ; t) \varphi(n) \rho(n),
\end{aligned}
$$


where the interchange in summation and integration is justifiable. For,

$$
\begin{aligned}
& \left|\sum_{n=0}^{\infty} \varphi(n) \rho(n) \int_{0}^{\infty} e^{-t u} L_{n}^{\alpha}(u) d \Omega(u)\right| \\
& \leq \frac{1}{\Gamma(\alpha+1)} \sum_{n=0}^{\infty}|\varphi(n)| \int_{0}^{\infty} e^{-t u} e^{u / 2} d \Omega(u)<\infty
\end{aligned}
$$

where we have used the well-known inequality

$$
\left|L_{n}^{\alpha}(x)\right| \leq \frac{e^{x / 2}}{\rho(n) \Gamma(\alpha+1)} .
$$

See $[4,(14)$, p. 207]. The result then follows by appealing to Corollary 3.2.

4. Examples of the inversion formula. The following examples illustrate Theorem 3.1.

I. We have, by [2, 3., p. 202],

$$
u(n, t)=e^{-t x} L_{n}^{\alpha}(x)=\sum_{m=0}^{\infty} g(n, m ; t) L_{m}^{\alpha}(x) \rho(m) .
$$

It follows that

$$
\begin{aligned}
& \lim _{t \rightarrow 0^{+}} \sum_{k=0}^{n} \frac{\Gamma(n+\alpha+1)}{k !(n-k) ! \Gamma(k+\alpha+1)} \frac{\partial^{k}}{\partial t^{k}}\left(e^{-t x}\right) \\
& =\lim _{t \rightarrow 0^{+}} \sum_{k=0}^{n} \frac{(-1)^{k} \Gamma(n+\alpha+1)}{k !(n-k) ! \Gamma(k+\alpha+1)} e^{-t x} x^{k} \\
& =L_{n}^{\alpha}(x),
\end{aligned}
$$

as predicted by the theorem.

II. Let

$$
\begin{aligned}
u(n, t) & =\frac{t^{n}}{(1+t)^{n+\alpha+1}} e^{-t x /(1+t)} L_{n}^{\alpha}\left(\frac{x}{t(1+t)}\right) \\
& =\sum_{m=0}^{\infty} g(n, m ; t) \frac{(-x)^{m}}{m !} \rho(m),
\end{aligned}
$$

where in $\left[\mathbf{2}, 4\right.$., p. 202] $\phi(m)$ should be given as $(-x)^{m} / m$ !. Then we have

$$
\begin{aligned}
\lim _{t \rightarrow 0^{+}} \sum_{k=0}^{n} & \frac{\Gamma(n+\alpha+1)}{k !(n-k) ! \Gamma(k+\alpha+1)} \frac{\partial^{k}}{\partial t^{k}}\left(\frac{1}{(1+t)^{\alpha+1}} e^{-t x /(1+t)}\right) \\
\quad= & \sum_{k=0}^{n} \frac{\Gamma(n+\alpha+1)(-1)^{k}}{k !(n-k) ! \Gamma(k+\alpha+1)} \sum_{l=0}^{k} \frac{\Gamma(k+\alpha+1)}{\Gamma(l+\alpha+1)}\left(\begin{array}{c}
k \\
l
\end{array}\right) x^{l} \\
& =\sum_{l=0}^{n} \frac{\Gamma(n+\alpha+1) x^{l}}{l !(n-l) ! \Gamma(l+\alpha+1)} \sum_{k=l}^{n} \frac{(n-l) !(-1)^{k}}{(k-l) !(n-k) !} \\
& =(-x)^{n} / n !
\end{aligned}
$$

the desired inversion. 
III. Let

$$
u(n, t)=\frac{1}{(1-x)^{\alpha+1}} g\left(n ; t+\frac{x}{1-x}\right)=\sum_{m=0}^{\infty} g(n, m ; t) x^{m} .
$$

See $[2,5 .$, p. 202]. Then we find that

$$
\begin{aligned}
\lim _{t \rightarrow 0^{+}} \sum_{k=0}^{n} & \frac{\Gamma(n+\alpha+1)}{k !(n-k) ! \Gamma(k+\alpha+1)} \frac{\partial^{k}}{\partial t^{k}}\left[\frac{1}{(1-x)^{\alpha+1}} g\left(0 ; t+\frac{x}{1-x}\right)\right] \\
& =\lim _{t \rightarrow 0^{+}} \sum_{k=0}^{n} \frac{\Gamma(n+\alpha+1)}{k !(n-k) ! \Gamma(k+\alpha+1)} \frac{(-1)^{k} \Gamma(k+\alpha+1)(1-x)^{k}}{(1+t(1-x))^{k+\alpha+1}} \\
& =x^{n} / \rho(n)
\end{aligned}
$$

as expected.

Theorem 3.4 may be illustrated by the following example.

Let

$$
\widehat{\varphi}(x)=e^{-t x}=\sum_{n=0}^{\infty} g(n ; t) L_{n}^{\alpha}(x) \rho(n) .
$$

See $[\mathbf{2}, 3 .$, p. 202 with $n=0]$. Then

$$
R(s)=\int_{0}^{\infty} e^{-t x} e^{-s x} d \Omega(x)=\frac{\Gamma(\alpha+1)}{(s+t+1)^{\alpha+1}}
$$

and

$$
R^{(k)}(0)=(-1)^{k} \Gamma(k+\alpha+1) /(t+1)^{k+\alpha+1}
$$

It follows that

$$
\begin{aligned}
\lim _{s \rightarrow 0^{+}} \sum_{k=0}^{n} \frac{\Gamma(n+\alpha+1)}{k !(n-k) ! \Gamma(k+\alpha+1)} R^{(k)}(s) & =\sum_{k=0}^{n} \frac{(-1)^{k} \Gamma(n+\alpha+1)}{k !(n-k) !(t+1)^{k+\alpha+1}} \\
& =\frac{\Gamma(n+\alpha+1)}{n !(t+1)^{\alpha+1}} \sum_{k=0}^{n}\left(\begin{array}{l}
n \\
k
\end{array}\right)\left(-\frac{1}{t+1}\right)^{k} \\
& =\frac{\Gamma(n+\alpha+1)}{n !} \frac{t^{n}}{(1+t)^{n+\alpha+1}} \\
& =g(n ; t),
\end{aligned}
$$

as the theorem stated.

5. Representation. We now establish a representation theorem for the Poisson-Laguerre transform.

THEOREM 5.1. A necessary and sufficient condition that

$$
u(n, t)=\sum_{m=0}^{\infty} g(n, m ; t) \varphi(m) \rho(m) ; \quad 0<t<\sigma,
$$

with $\varphi(m) \geq 0$, and, for some $t_{0}>0$,

$$
\sum_{m=0}^{\infty} u\left(m, t_{0}\right)<\infty
$$


is that

$$
u(n, t)=\int_{0}^{\infty} e^{-t x} L_{n}^{\alpha}(x) \widehat{\varphi}(x) d \Omega(x),
$$

where

$$
\widehat{\varphi}(x)=\sum_{m=0}^{\infty} L_{m}^{\alpha}(x) \varphi(m) \rho(m)
$$

and

$$
\sum_{m=0}^{\infty} \varphi(m)<\infty .
$$

ProOF. Suppose, that for $\varphi(m) \geq 0$,

$$
u(n, t)=\sum_{m=0}^{\infty} g(n, m ; t) \varphi(m) \rho(m)
$$

with (5.2) holding. Then, since, by [2, (4.4), p. 205],

$$
\sum_{m=0}^{\infty} g(n, m ; t)=\frac{1}{\rho(n)},
$$

we have

$$
\begin{aligned}
\sum_{n=0}^{\infty} u\left(n, t_{0}\right) & =\sum_{n=0}^{\infty} \sum_{m=0}^{\infty} g\left(n, m ; t_{0}\right) \varphi(m) \rho(m) \\
& =\sum_{m=0}^{\infty} \varphi(m)<\infty .
\end{aligned}
$$

It follows that the series

$$
\sum_{m=0}^{\infty} L_{m}^{\alpha}(x) \varphi(m) \rho(m)
$$

converges absolutely and defines a function $\varphi^{\widehat{\gamma}}(x)$. Now consider

$$
\begin{aligned}
\int_{0}^{\infty} e^{-t x} & L_{n}^{\alpha}(x) \varphi^{-}(x) d \Omega(x) \\
& =\int_{0}^{\infty} e^{-t x} L_{n}^{\alpha}(x) d \Omega(x) \sum_{m=0}^{\infty} L_{m}^{\alpha}(x) \varphi(m) \rho(m) \\
& =\sum_{m=0}^{\infty} \varphi(m) \rho(m) \int_{0}^{\infty} e^{-t x} L_{n}^{\alpha}(x) L_{m}^{\alpha}(x) d \Omega(x) \\
& =\sum_{m=0}^{\infty} g(n, m ; t) \varphi(m) \rho(m)=u(n, t),
\end{aligned}
$$


where the interchange in summation and integration is valid. For, appealing to (3.9), and taking note of (5.8), we have

$$
\begin{aligned}
\mid \sum_{m=0}^{\infty} \varphi(m) & \rho(m) \int_{0}^{\infty} e^{-t x} L_{n}^{\alpha}(x) L_{m}^{\alpha}(x) d \Omega(x) \mid \\
\leq & \sum_{m=0}^{\infty} \varphi(m) \int_{0}^{\infty} \frac{e^{-t x} x^{\alpha}}{\rho(n)[\Gamma(\alpha+1)]^{2}} d x<\infty
\end{aligned}
$$

The necessity of the conditions is thus established.

To prove sufficiency assume that

$$
u(n, t)=\int_{0}^{\infty} e^{-t x} L_{n}^{\alpha}(x) \hat{\varphi}(x) d \Omega(x)
$$

with (5.4) and (5.5) holding. An appeal to (5.8) yields (5.2). Moreover, applying to (5.1) the calculation of (5.10), we obtain the desired representation (5.1) and the theorem is proved.

6. Examples of the representation theorem. We may illustrate Theorem 5.1 by taking

$$
\varphi(m)=g(m ; a)
$$

a nonnegative function. Then, by $\left[2,(4.16)\right.$, p. 207, with $\left.m=0, t_{1}=a, t_{2}=t\right]$, we have

$$
u(n, t)=\sum_{m=0}^{\infty} g(n, m ; t) g(m ; a) \rho(m)=g(n ; t+a)
$$

where, by $[2,(4.4)$, p. 205, with $n=0]$,

$$
\sum_{n=0}^{\infty} g(n ; t+a)=\Gamma(\alpha+1)
$$

Thus (5.2) holds for all $t$. Further, we have

$$
g(n ; t+a)=\int_{0}^{\infty} e^{-(t+a) x} L_{n}^{\alpha}(x) d \Omega(x)
$$

with

$$
\sum_{m=0}^{\infty} g(m ; a)=\Gamma(\alpha+1)
$$

Also (5.4) holds since, by $[2,(2.30)$, p. 201, with $m=0, t=a]$, we have $\widehat{\varphi}(x)=$ $e^{-a x}$.

Since we know, by $[2$, Theorem 10.1, p. 223], that $u(n, t)$ is representable by a Poisson-Laguerre transform of a nonnegative function $\varphi$ if and only if $u(n, t)$ is a nonnegative Laguerre temperature, Theorem 5.1 deals with a subclass of the nonnegative Laguerre temperatures. We note, for example, that, for

$$
\varphi(m)=m / \rho(m)
$$


we have, using [2, 2., p. 202],

$$
u(n, t)=\sum_{m=0}^{\infty} m g(n, m ; t)=\frac{n+t(\alpha+1)}{\rho(n)} .
$$

Condition (5.2) fails for any $t$. Thus although $u(n, t)$ has a Poisson-Laguerre representation (5.1), it fails to have the representation (5.3), as the hypotheses of the theorem are not satisfied.

\section{REFERENCES}

1. F. M. Cholewinski and D. T. Haimo, Integral representations of solutions of the generalized heat equation, Illinois J. Math. 10 (1966), 623-638.

2. __ Laguerre temperatures, Proc. Conf. Orthogonal Expansions and Their Continuous Analogues (D. T. Haimo, ed.), Southern Illinois Univ. Press, Carbondale, 1968, pp. 197-226.

3. I Inversion of the reduced Poisson- Hankel transform, J. Analyse Math. 25 (1972), 323-343.

4. A. Erdélyi et al, Higher transcendental functions, McGraw-Hill, New York, 1953.

5. D. T. Haimo, The reduced dual Poisson-Laguerre transform, J. Math. Anal. Appl. (to appear).

6. D. V. Widder, The role of the Appell transformation in the theory of heat conduction, Trans. Amer. Math. Soc. 109 (1963), 121-134.

7. Math. Soc. 4 (1964), 1-14.

8. _ , The Laplace transform, Princeton Univ. Press, Princeton, N.J., 1941.

Department of Mathematical Sciences, University of Missouri, St. Louis, MISSOURI 63121 (Current address)

School of Mathematics, Institute for Advanced Study, Princeton, NeW JERSEY 08540 